ORIGINAL ARTICLE

\title{
Acceptability of identification bracelets for hospital inpatients
}

\author{
A Cleopas, V Kolly, P A Bovier, P Garnerin, T V Perneger
}

See editorial commentary, p 329

Qual Saf Health Care 2004;13:344-348. doi: 10.1136/qshc.2004.010496

See end of article for authors' affiliations

Correspondence to:

A Cleopas, Quality of Care

Unit, Geneva University

Hospitals, Geneva,

Switzerland; agatta.

cleopas@hcugc.ch

Accepted for publication

26 July 2004
Objective: To evaluate whether hospitalised patients would agree to wear an identification bracelet and whether patient acceptability is improved by more detailed explanations or by using a code instead of a name on the bracelet.

Design: Patient survey that tested two variables in a randomised factorial design. Explanations about identification bracelets were given (a) with or without examples of situations where patient identification may be important, and (b) with the patient name or an anonymous code appearing on the bracelet.

Setting: Swiss teaching hospital where wearing of identification bracelets was not systematic.

Participants: Adult patients discharged from hospital $(n=1411)$.

Main outcome measures: Patients' responses to the questions: (a) should the hospital introduce a compulsory identification bracelet? and (b) would the patient agree to wear such a bracelet?

Results: Globally, $83.9 \%$ of patients thought that the hospital should introduce bracelets and $90.2 \%$ stated that they would agree to wear one. Providing examples increased support for both the hospital policy $(87.9 \% \vee 79.2 \%, p<0.001)$ and personal acceptance $(92.2 \% v 88.1 \%, p=0.015)$. Whether or not the bracelet carried the patient's name or an anonymous code did not influence patient choice.

Conclusions: The majority of patients were in favour of wearing an identification bracelet during their hospital stay. This proportion increased significantly when an explanation based on examples of the consequences of incorrect patient identification had been provided.
$\mathrm{P}$ atient identification is crucial for patient safety. ${ }^{1-4}$ The Joint Commission on Accreditation of Healthcare Organizations (JCAHO) reported that incorrect patient identification is involved in $13 \%$ of medical errors in surgery ${ }^{3}$ and $67 \%$ of transfusion errors. ${ }^{45}$ A recent case study of an invasive procedure undertaken on the wrong person showed that, of 17 discrete errors committed during this incident, 10 were related to the absence of a standardized protocol for patient identification. ${ }^{146}$ Correct patient identification has therefore become a priority. ${ }^{367}$ For instance, "improvement of the identification of the patient" is the first Patient National Safety Goal for 2004 in the United States. ${ }^{7} 8$

To reduce patient identification errors, many hospitals provide patients with identification wristbands to be worn during the entire hospital stay. ${ }^{4}{ }^{10}$ While use of patient identification bracelets is almost universal in North American hospitals, this procedure is not routine in Switzerland and in most European countries. At our hospital, at the time of this study, an identification bracelet was given to selected patients only, such as those suffering from dementia or those about to undergo surgery. In preliminary discussions about the feasibility of generalising identification bracelets to all patients at our hospital, some hospital staff considered this measure to be demeaning to patients and believed that they would refuse to wear such bracelets. Similar concerns were noted by others. ${ }^{11}$ What patients themselves thought was unknown.

A study was undertaken to ascertain whether patients would agree to wear an identification bracelet during their hospital stay. We also tested whether acceptability would be improved by more detailed explanations or by using an anonymous code instead of the patient's name on the bracelet.

\section{METHODS}

\section{Study design and setting}

A cross sectional study was conducted at the Geneva University Hospitals as part of the annual patient opinion survey. The study was exempt from formal review by the hospital research ethics committee because, as a quality improvement project, it involved minimal risk to participants. The Geneva University Hospitals system includes an acute care hospital, a psychiatric hospital, a geriatric hospital and a long term care facility totalling 2200 beds and more than 45600 inpatient admissions in 2002.

\section{Patients, data collection, and questionnaire}

The participants were all adult patients ( 18 years and over) who were discharged alive between 15 September and 15 October 2002, identified from administrative databases. Of 2551 subjects, five with an unspecified address were excluded as well as 155 patients transferred to other hospitals, 21 hospitalised for less than 24 hours, 92 who were discharged for a second time during this period, and three mothers of stillborn children. The patient opinion survey was therefore sent to 2275 individuals.

A survey package including a cover letter, the questionnaire, and a business reply envelope was sent to patients' homes 1-2 months after their discharge. Follow up mailings were sent after 1 and 2 months to non-respondents, as in other patient surveys at our hospital. ${ }^{12}$

The questionnaire included a front page with explanations about the survey and questions about exclusion criteria (does not speak French, difficulty reading or writing, too sick to answer questionnaire), a patient satisfaction instrument (Picker Patient Experience Instrument ${ }^{13}$ ), an additional question on the feeling of security during the hospital stay, patient demographic variables (age, sex, nationality, level of education) and questions regarding the occurrence of complications and problems during the stay in hospital. These problems were classified into two groups:

- incidents possibly related to identification errors (received a drug that was not intended for you; had an allergic reaction to a drug; tests repeated unnecessarily by 


\section{Box 1 Types of explanations and questions}

Example/anonymous code

Incorrect patient identification may cause problems in a hospital-for example, a patient can receive a treatment that was not intended for him/her or, if a patient faints in a corridor of the hospital and nobody knows him/her, it is useful to have quick access to his/her medical file. To avoid these problems some hospitals have introduced the compulsory wearing of an identification bracelet to allow fast and simple identification of each patient. This bracelet bears a unique anonymous code.

No example/name

Incorrect patient identification may cause problems in a hospital. To avoid these problems some hospitals have introduced the compulsory wearing of an identification bracelet to allow fast and simple identification of each patient. This bracelet bears the name of the patient and that of the ward where he/she is hospitalised.

Questions

- Should the Geneva University Hospitals introduce the compulsory wearing of such a bracelet for all patients? ("Yes"/ /"No")

- If not, please specify why (open field)

- If such a system was implemented at the Geneva University Hospitals, would you agree to wear such a bracelet during your next hospitalisation? ("Yes"/ "No")

- If you do not agree, please explain why (open field)

mistake; confused with another patient during a test or procedure; medical file or your $x$ rays not available when needed); and

- other incidents.

\section{Acceptability of identification bracelet}

In addition, participants were invited to express their opinions about wearing an identification bracelet during their hospital stay. We tried to determine whether the acceptability of a bracelet would improve when examples of situations in which patient identification was a concern were given, or when the identifier on the bracelet was an anonymous code rather than the patient's name. Participants were randomly given one of the following four types of explanation, using a factorial design:
- examples/name;

- examples/code;

- no examples/name; and

- no examples/code.

The explanation was followed by four questions (box 1).

These questions, as well as the various versions of the scenario, were developed in house and pre-tested with 34 inpatients to ensure that they were easily understood.

\section{Study variables}

The dependent variables were the support for a hospital policy providing all patients with an identification bracelet and personal agreement to wear such a bracelet during a future hospital admission.

The main independent variables were the two experimental factors - that is, illustration by examples of why being unable to identify a patient may cause problems, and name versus code on the bracelet. Other independent variables included patient age, sex, length of stay, occurrence of any incidents during the hospital stay (incidents possibly related to patient identification versus others), patients' feeling of having been treated in safe conditions ("Do you think that you were treated in safe conditions?": "yes, completely" $v$ "yes, in part" and "no"), and their overall evaluation of the care received at the hospital ("excellent" or "very good" $v$ "good" or "fair" or "poor").

\section{Statistical analysis}

Cross-tabulations and $\chi^{2}$ tests were used to study, firstly, how experimental factors and then how patient characteristics and hospital stay experience influence the dependent variables. We also built a logistic regression model of acceptability of identification bracelets among hospitalised patients with the two experimental variables and all other independent variables as covariates. In all analyses $p$ values of $<0.05$ were considered to be statistically significant. SPSS version 11.0 was used to perform statistical analysis.

\section{Analysis of open comments}

To better understand reasons for refusal we transcribed all answers to the open questions about reasons for not supporting generalised wearing of the identification bracelet. These answers were classified into common themes. To analyse the comments a coding grid with seven categories was elaborated after the first reading of the answers: uselessness, selective use of bracelet, refusal of the obligation, lack of respect, need to improve work of staff, aspect of bracelet, and patient responsibility. Each answer was coded

Table 1 Support for identification bracelet among former patients

\begin{tabular}{|c|c|c|c|c|}
\hline \multirow{2}{*}{$\begin{array}{l}\text { Variable } \\
\text { Overall }\end{array}$} & \multirow{2}{*}{$\begin{array}{l}\mathbf{N} \\
1289\end{array}$} & $\begin{array}{l}\text { Hospital should introduce } \\
\text { compulsory wearing of } \\
\text { identification bracelet } \\
\text { (\% agree) }\end{array}$ & \multicolumn{2}{|c|}{$\begin{array}{l}\text { Would wear such a bracelet } \\
\text { during a future } \\
\text { hospitalisation } \\
\text { (\% agree) }\end{array}$} \\
\hline & & 83.6 & 90.2 & \\
\hline \multicolumn{5}{|c|}{$\begin{array}{l}\text { Examples of situations in which patient } \\
\text { identification was a concern }\end{array}$} \\
\hline Given & 648 & $(p<0.001)$ & 92.2 & $(p=0.015)$ \\
\hline Not given & 641 & 79.2 & 88.1 & \\
\hline \multicolumn{5}{|c|}{ Means of patient identification on bracelet } \\
\hline Patient name & 626 & $(p=0.92)$ & 90.2 & $(p=0.98)$ \\
\hline Anonymous code & 636 & 83.5 & 90.2 & \\
\hline
\end{tabular}


Table 2 Free comments contributed by patients who were not in favour of the introduction of a hospital policy of a compulsory identification bracelet and who did not agree to wear such a bracelet

\begin{tabular}{|c|c|}
\hline Category & Patients' comments \\
\hline Uselessness & $\begin{array}{l}\mathrm{N}=55 \\
\text { Example: "The responsibility and competences of the } \\
\text { medical staff being excellent, wearing a bracelet is } \\
\text { useless." }\end{array}$ \\
\hline $\begin{array}{l}\text { Selective use of } \\
\text { bracelet }\end{array}$ & $\begin{array}{l}\mathrm{N}=64 \\
\text { Example: "The bracelet is necessary for certain } \\
\text { patients (with high risk), but it should not be } \\
\text { generalised." }\end{array}$ \\
\hline Lack of respect & $\begin{array}{l}\mathrm{N}=59 \\
\text { Example: "I refuse to be an anonymous patient who } \\
\text { must wear a label to be recognised." }\end{array}$ \\
\hline $\begin{array}{l}\text { Refusal of } \\
\text { obligation }\end{array}$ & $\begin{array}{l}n=16 \\
\text { Example: "I don't like the term 'compulsory', } \\
\text { everyone must have the right to choose." }\end{array}$ \\
\hline $\begin{array}{l}\text { Patient } \\
\text { responsibility }\end{array}$ & $\begin{array}{l}n=24 \\
\text { Example: "The patients who know their identity do } \\
\text { not need any identification bracelet." }\end{array}$ \\
\hline $\begin{array}{l}\text { Need to improve } \\
\text { work of staff }\end{array}$ & $\begin{array}{l}n=14 \\
\text { Example: "With staff in a sufficient number, I think } \\
\text { that these problems of identification would be quasi } \\
\text { non-existent." }\end{array}$ \\
\hline Aspect of bracelet & $\begin{array}{l}n=10 \\
\text { Example: "I would agree only if the bracelet is } \\
\text { discreet." }\end{array}$ \\
\hline
\end{tabular}

by two readers and discrepancies were resolved by consensus. Because the patients' comments to these two questions were very similar, we analysed them together.

\section{RESULTS}

\section{Characteristics of patients}

Of 2275 patients to whom the questionnaires were sent, 44 had died, 77 had an invalid address, 48 did not speak French, and 93 were not able to answer; 1411 of the remaining 2013 patients $(70 \%)$ returned the questionnaire.

The majority of respondents were women ( $n=793,56.2 \%)$ and Swiss nationals $(\mathrm{n}=945,67 \%)$. The mean (SD) age was
55.2 (20.1) years (quartiles 37-56-73). Patients had been discharged from the departments of surgery $(n=422$, $29.9 \%)$, internal medicine $(n=310,22 \%)$, gynaecology/ obstetrics $(\mathrm{n}=270,19.1 \%)$, clinical neurosciences and dermatology $(n=220,15.6 \%)$, geriatrics $(n=104,7.4 \%)$, and psychiatry $(n=79,5.6 \%)$. Six patients had missing information on this variable. The mean (SD) length of stay was 12.1 (18.3) days (quartiles 4-6-13; range 2-224 days) and most patients had left the hospital for their home $(\mathrm{n}=1328,94.1 \%)$.

\section{Acceptability of an identification bracelet}

Of the 1411 survey respondents, 1289 (91\%) answered one or both of the closed format questions about the acceptability of an identification bracelet. Those who did not answer these questions were slightly older than those who did (mean age 64 years $v 54$ years, $\mathrm{p}<0.001)$, tended to be women $(69 \%$ non-respondents $v 55 \%$ respondents, $\mathrm{p}=0.003$ ), and had longer hospital stays (mean 18 days $v 12$ days, $\mathrm{p}<0.001$ ).

The question "Should the Geneva University Hospitals introduce the compulsory wearing of an identification bracelet?" was answered by 1262 individuals of whom 1055 $(83.6 \%)$ answered in the affirmative (table 1). Providing examples significantly increased the support for this policy, but whether the bracelet bore the name or a code was of no importance.

The second closed format question ("Would you agree to wear such a bracelet during a future hospitalisation?") was answered by 1265 patients and 1141 (90\%) agreed (table 1). Again, providing examples improved the acceptability of the bracelet, but the name or the code as the identifier had no effect.

\section{Open comments}

Among patients who stated that they would either oppose the hospital policy or refuse to wear the bracelet $(n=207), 138$ provided a total of 242 explanations to justify their answers. The main explanations were that the bracelet is useless, that wearing of an identification bracelet should depend on the patient's situation, and that forcing patients to wear an identification bracelet showed lack of respect (table 2).

\begin{tabular}{|c|c|c|c|c|c|}
\hline \multirow{2}{*}{$\begin{array}{l}\text { Variable } \\
\text { Sex }\end{array}$} & & \multicolumn{2}{|c|}{$\begin{array}{l}\text { Hospital policy } \\
\text { (\% agree) }\end{array}$} & \multicolumn{2}{|c|}{$\begin{array}{l}\text { Personal acceptance } \\
\text { (\% agree) }\end{array}$} \\
\hline & & & & & \\
\hline Women & 694 & 84.3 & $(p=0.46)$ & 92.2 & $(p=0.99)$ \\
\hline Men & 568 & 82.7 & & 92.2 & \\
\hline \multicolumn{6}{|l|}{ Age (years) } \\
\hline $18-44$ & 473 & 83.5 & $(p=0.94)$ & 91.7 & $(p=0.94)$ \\
\hline $45-64$ & 347 & 84.1 & & 89.2 & \\
\hline $65-98$ & 442 & 83.3 & & 89.4 & \\
\hline \multicolumn{6}{|l|}{ Length of stay (days) } \\
\hline $2-10$ & 896 & 84.5 & $(p=0.28)$ & 90.6 & $(p=0.26)$ \\
\hline $11-30$ & 273 & 82.4 & & 90.4 & \\
\hline$>30$ & 93 & 78.5 & & 85.4 & \\
\hline \multicolumn{6}{|l|}{ Felt treated in complete safety } \\
\hline Yes & 835 & 83.7 & $(p=0.78)$ & 90.5 & $(p=0.75)$ \\
\hline No & 206 & 84.6 & & 89.8 & \\
\hline \multicolumn{6}{|l|}{ Care received at hospital } \\
\hline Excellent/very good & 643 & 83.6 & $(p=0.94)$ & 90.7 & $(p=0.51)$ \\
\hline Good/poor/fair & 398 & 83.8 & & 89.6 & \\
\hline \multicolumn{6}{|l|}{$\begin{array}{l}\text { Incidents possibly related to } \\
\text { identification errors }\end{array}$} \\
\hline None & 798 & 84.0 & $(p=0.92)$ & 90.9 & $(p=0.32)$ \\
\hline Any & 239 & 83.7 & & 88.8 & \\
\hline \multicolumn{6}{|l|}{ Other incidents } \\
\hline None & 684 & 85.4 & $(p=0.24)$ & 92.4 & $(p=0.014)$ \\
\hline Any & 368 & 82.6 & & 87.8 & \\
\hline
\end{tabular}


Table 4 Associations of independent variables with acceptability of the hospital policy of systematic bracelets and personal acceptance to wear an identification bracelet (multiple logistic regression model)

\begin{tabular}{|c|c|c|c|}
\hline & & \multirow{2}{*}{$\begin{array}{l}\text { Hospital policy } \\
\text { Odds ratio }(95 \% \mathrm{Cl})\end{array}$} & \multirow{2}{*}{$\frac{\text { Personal acceptance }}{\text { Odds ratio }(95 \% \mathrm{Cl})}$} \\
\hline & & & \\
\hline $\begin{array}{l}\text { Examples of situations in which patient } \\
\text { identification was a concern }\end{array}$ & Given $v$ not given & $1.8(1.3$ to 2.6$)$ & 1.7 (1.1 to 2.8$)$ \\
\hline Means of patient identification on bracelet & Anonymous code $v$ patient name & $1.2(0.8$ to 1.7$)$ & $1.1(0.7$ to 1.7$)$ \\
\hline Sex & Male $v$ female & $1.1(0.7$ to 1.5$)$ & $1.1(0.7$ to 1.7$)$ \\
\hline \multirow[t]{2}{*}{ Age (years) } & $45-64$ > $18-44$ & $1.1(0.7$ to 1.6$)$ & $0.7(0.4$ to 1.2$)$ \\
\hline & $65-98$ v 18-44 & $1.2(0.8$ to 2.0$)$ & $0.8(0.4$ to 1.4$)$ \\
\hline \multirow[t]{2}{*}{ Length of stay (days } & $11-30$ $2-10$ & $1.3(0.8$ to 2.2$)$ & $1.5(0.8$ to 2.7$)$ \\
\hline & $>30 \vee 2-10$ & $0.6(0.3$ to 1.2$)$ & $1.1(0.4$ to 2.5$)$ \\
\hline Felt treated in complete safety & No ryes & $1.0(0.6$ to 1.8$)$ & $0.9(0.5$ to 1.7$)$ \\
\hline Care received at the hospital & Excellent/very good $v$ good/poor/fair & $0.9(0.6$ to 1.4$)$ & $0.9(0.6$ to 1.6$)$ \\
\hline Incidents possibly related to identification errors & Any $v$ none & $1.0(0.7$ to 1.6$)$ & $1.1(0.6$ to 2.0$)$ \\
\hline Other incidents & Any $v$ none & $0.8(0.5$ to 1.2$)$ & $0.6(0.3$ to 0.8$)$ \\
\hline
\end{tabular}

\section{Subgroup comparisons}

The age and sex of the patients were not related to the acceptability of the bracelet, nor were the length of stay, the feeling of being treated in complete safety, and the global evaluation of care received at the hospital (table 3 ).

Somewhat surprisingly, patients who reported no incidents during their stay in hospital were more supportive of compulsory identification bracelets than patients who did (table 3). This difference was seen particularly if the incident was unrelated to patient identification errors.

In logistic regression analysis, support for the hospital policy of having all patients wear identification bracelets was associated only with the provision of examples. Assent to wear an identification bracelet during a forthcoming hospital admission was also increased by the provision of examples but was lower if the patient had reported an incident unrelated to patient identification (table 4).

\section{DISCUSSION}

Most patients $(83.9 \%)$ supported a policy to introduce the compulsory wearing of an identification bracelet, and $90 \%$ would agree to wear such a bracelet during a forthcoming hospital admission. Overall, these results indicate that assumptions among hospital staff that patients would reject identification bracelets were unjustified. More generally, these results suggest that patients may be an underused resource for setting effective hospital policies. ${ }^{14}{ }^{15}$

Nevertheless, a minority of patients expressed objections to wearing such a bracelet. Whether these patients would refuse to wear a bracelet in real life situations is unclear. Handling refusals may require special policies. Inconsistent application of identification bracelets may hamper their effectiveness in reducing errors.

\section{Key messages}

- In a hospital where wearing of identification bracelets was not systematic, most of patients were in favour of wearing an identification bracelet during their hospital stay.

- Illustrating the explanation with examples of the consequences of incorrect patient identification increased the proportion of patients who agreed to wear an identification bracelet.

- Patients' opinions may be a resource for the development of effective hospital policies.
Illustrating the explanation about identification bracelets with examples increased patient support for this measure. This suggests that, when appropriate explanations regarding hospital policies are given, patient understanding and hence probable compliance with the policy will increase.

Whether the identifier was nominative or anonymous did not appear to influence bracelet acceptability. Thus, those who objected were not primarily concerned about unwitting disclosure of their name. This was confirmed by the analysis of the reasons for refusing the bracelet: patients did not mention fearing a breach of privacy.

We had expected greater support for identification bracelets from patients who suffered an adverse event during their own hospital stay. What we found was the opposite: patients who reported no incidents were more likely to favour bracelets. Possibly, persons who experienced no incidents during their hospital stay have greater confidence in the hospital and would more readily accept safety measures suggested by the hospital. Another possibility is that this was a chance finding.

The use of identification bracelets for hospitalised patients may in itself cause errors. An analysis of identification errors in 712 hospitals in North America estimated that $5.5 \%$ of the errors were due to the identification bracelet-for example, absence of the bracelet, conflict between data sources, incorrect information, illegible data. ${ }^{16}$ However, effective use of bracelets may be subject to a learning curve: wristband errors identified by phlebotomists at 217 US hospitals decreased by more than half over a period of 2 years. ${ }^{10}$ Other authors suggest that standardised protocols should be established for the verification of identification bracelets and other procedures related to patient identity. ${ }^{6}$ Such protocols should be applied to all willing patients by all members of the healthcare team.

The main limitation of this study is that the responders reacted to a hypothetical situation. We are not sure if patients will respond in the same manner in a real life situation. Another limitation is the possibility of selection bias. Patients who did not return the questionnaire may have different attitudes toward the identification bracelets.

In conclusion, the introduction of the generalised wearing of an identification bracelet during a hospital stay should be acceptable to most patients at our hospital. As with all healthcare interventions, the implementation of this safety measure will require careful monitoring of possible undesirable effects.

\section{Authors' affiliations}

A Cleopas, V Kolly, T V Perneger, Quality of Care Unit, Geneva University Hospitals, Geneva, Switzerland 
P A Bovier, Department of Community Medicine, Geneva University Hospitals, Geneva, Switzerland

P Garnerin, Division of Anaesthesiology, Geneva University Hospitals, Geneva, Switzerland

\section{REFERENCES}

1 Chassin MR, Becher EC. The wrong patient. Ann Intern Med 2002:136:826-33.

2 Serig DI. Radiopharmaceutical misadministration: what's wrong? In: Bogner MS, eds. Human error in medicine. Hove, UK: Lawrence Erlbaum Associates, 1994.

3 Beyea SC. Patient identification-a crucial aspect of patient safety. AORN J 2003;78:478-82

4 ECRI. Patient identification. Risk analysis. Risk and quality management strategies 16. Healthcare Risk Control. 2003;Supplement A: 1-11, http:// www.ecri.org/PatientSafety/RiskQual 16.pdf (accessed 16 October 2003)

5 Sentinel Event Statistics (online). Root causes of transfusion events, Joint Commission on Accreditation for Healthcare Organizations (JCAHO), 2002.http://www.jcaho.org/accredited+organizations/ambulatory+care/ sentinel+events/rc+transfusion+events.htm (accessed 12 January 2004).

6 Beyea SC. Systems that reduce the potential for patient identification errors. Aorn J 2002:76:510-12.

7 Beyea SC. The National Patient Safety Goals and their implications for perioperative nurses. AORN J 2003;77:1241-5.
8 Joint Commission on Accreditation for Healthcare Organizations (JCAHO) National Patient Safety Goals, http://www.jcaho.org/ accredited+organizations/patient+safety/04+npsg/index.htm (accessed 27 October 2003).

9 Anon. Nouveau bracelet d'identification pour le patient. Bulletin d'Information Cliniques 2003; 103:29.

10 Howanitz PJ, Renner SW, Walsh MK. Continuous wristband monitoring over 2 years decreases identification errors. A College of American Pathologists QTracks Study. Arch Pathol Lab Med 2002;126:809-15.

11 Ellenberg E. Acceptability, social imagery and the power of technology: example of wearing an identification bracelet at the hospital (in French). Esprit Critique 2004;6:155-63.

12 Perneger TV, Kossovsky MP, Cathieni F, et al. A randomised trial of four patient satisfaction questionnaires. Med Care 2003:41:1343-52.

13 Jenkinson C, Coulter A, Bruster S, et al. Patient's experiences and satisfaction with health care: results of a questionnaire study of specific aspects of care. Qual Saf Health Care 2002;11:335-9.

14 Keslon M. Patient involvement in clinical governance. In: Lugon M, SeckerWalker J, eds. Advancing clinical governance. London UK: Royal Society of Medicine, 2001

15 Vincent CA, Coulter A. Patient safety: what about the patient? Qual Saf Health Care 2002;11:76-80.

16 Renner SW, Howanitz PJ, Bachner P. Wristband identification error reporting in 712 hospitals. A College of American Pathologists' Q-Probes Study of Quality Issues in Transfusion Practice. Arch Pathol Lab Med 1993; 1 17:573-7.

$\mathrm{ECHO}$

\section{Capturing the full story is essential for good care}

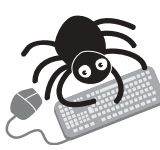

Please visit the Quality and Safety in Health Care website [www.qshc com] for a link to the full text of this article.
$\mathrm{T}$ he usefulness of electronic health records must be improved if patients are to be well managed, argues one clinician in England. The comment is timely as service providers to implement a national Integrated Care Record Service have just been appointed.

Patients sharing their perspective with their doctor often have better outcomes. So getting electronic health records to reflect the truest clinical picture is essential to ensuring good patient care. That is a challenge because doctors intuitively get their information from patients in the form of narratives and communicate with each other in this form too. Narratives allow doctors to refine their diagnosis as they unfold, but are not readily captured by current electronic systems. Structured, selective systems of capturing information simply do not compare.

Preserving the process of clinical reasoning is also paramount. This could best be achieved by developing systems which could cope with direct handwritten input-notes and sketches-and voice input by doctors themselves, preferably into a portable device. Many useful tools - diagnostic aids, best evidence, and local and national clinical guidelines - can be incorporated into the systems, but the knowledge structure must not intrude on the clinical reasoning process, and more research is needed to establish their true benefits. Retrieving and presenting the information usefully is just as much of a challenge.

"Clinicians need to be closely involved in ensuring that software for documenting patient encounters complements the way they work." It's now or never.

A Walsh, SH. BMJ 2004;328:1184-1187. 